\title{
p38/Sp1/Sp4/HDAC4/BDNF Axis Is a Novel Molecular Pathway of the Neurotoxic Effect of the Methylmercury
}

\author{
Natascia Guida ${ }^{1}$, Giusy Laudati ${ }^{2}$, Luigi Mascolo ${ }^{2}$, Valeria Valsecchi ${ }^{2}$, Rossana Sirabella ${ }^{2}$, \\ Carmine Selleri ${ }^{3}$, Gianfranco Di Renzo ${ }^{2}$, Lorella M. T. Canzoniero ${ }^{2,4}$ and \\ Luigi Formisano $^{2,4 *}$ \\ ${ }^{1}$ IRCCS SDN, Naples, Italy, ${ }^{2}$ Division of Pharmacology, Department of Neuroscience, Reproductive and Dentistry Sciences, \\ School of Medicine, "Federico II" University of Naples, Naples, Italy, ${ }^{3}$ Department of Medicine and Surgery, University of \\ Salerno, Salerno, Italy, ${ }^{4}$ Division of Pharmacology, Department of Science and Technology, University of Sannio, Benevento, \\ Italy
}

\section{OPEN ACCESS}

Edited by:

Natalia V. Gulyaeva,

Institute of Higher Nervous Activity

and Neurophysiology, Russian Academy of Sciences (RAS), Russia

Reviewed by:

Aurel Popa-Wagner

University of Rostock, Germany

George Smith,

Temple University School of Medicine

${ }^{*}$ Correspondence:

Luigi Formisano

cformisa@unisannio.it

Specialty section:

This article was submitted to

Neurodegeneration,

a section of the journal

Frontiers in Neuroscience

Received: 15 September 2016 Accepted: 04 January 2017 Published: 19 January 2017

Citation:

Guida N, Laudati G, Mascolo L, Valsecchi V, Sirabella R, Selleri C, Di

Renzo G, Canzoniero LMT and Formisano L (2017) p38/Sp1/Sp4/HDAC4/BDNF Axis Is a Novel Molecular Pathway of the Neurotoxic Effect of the Methylmercury. Front. Neurosci. 11:8 doi: 10.3389/fnins.2017.00008
The molecular pathways involved in methylmercury (MeHg)-induced neurotoxicity are not fully understood. Since pan-Histone deacetylases (HDACs) inhibition has been found to revert the neurodetrimental effect of $\mathrm{MeHg}$, it appeared of interest to investigate whether the pattern of HDACs isoform protein expression is modified during MeHg-induced neurotoxicity and the transcriptional/transductional mechanisms involved. SH-SY5Y neuroblastoma cells treated with $\mathrm{MeHg} 1 \mu \mathrm{M}$ for 12 and $24 \mathrm{~h}$ showed a significant increase of HDAC4 protein and gene expression, whereas the HDACs isoforms 1-3, 5, and 6 were unmodified. Furthermore, MeHg-induced HDAC4 increase was reverted when cells were transfected with siRNAs against specificity protein 1 (Sp1) and $\mathrm{Sp} 4$, that were both increased during MeHg exposure. Next we studied the role of extracellular-signal-regulated kinases 1/2 (ERK1/2), c-Jun N-terminal kinases (JNK), and p38 mitogen-activated protein kinases (MAPKs) in MeHg-induced increase of Sp1, $\mathrm{Sp} 4$, and HDAC4 expression. As shown by Western Blot analysis $\mathrm{MeHg}$ exposure increased the phosphorylation of p38, but not of ERK and JNK. Notably, when p38 was pharmacologically blocked, MeHg-induced Sp1, Sp4 protein expression, and HDAC4 protein and gene expression was reverted. In addition, MeHg exposure increased the binding of HDAC4 to the promoter IV of the Brain-derived neurotrophic factor (BDNF) gene, determining its mRNA reduction, that was significantly counteracted by HDAC4 knocking down. Furthermore, rat cortical neurons exposed to MeHg $(1 \mu \mathrm{M} / 24 \mathrm{~h})$ showed an increased phosphorylation of p38, in parallel with an up-regulation of Sp1, Sp4, and HDAC4 and a down-regulation of BDNF proteins. Importantly, transfection of siRNAs against p38, Sp1, Sp4, and HDAC4 or transfection of vector overexpressing BDNF significantly blocked MeHg-induced cell death in cortical neurons. All these results suggest that p38/Sp1-Sp4/HDAC4/BDNF may represent a new pathway involved in MeHg-induced neurotoxicity.

Keywords: MeHg, HDAC4, p38, Sp transcription factors, BDNF, neuronal cell death 


\section{INTRODUCTION}

Exposure to MeHg is detrimental, particularly for the developing brain (Ceccatelli et al., 2013). Indeed prenatal poisoning with high dose of $\mathrm{MeHg}$ causes mental retardation and cerebral palsy (Myers and Davidson, 1998). Regarding the possible relationship between $\mathrm{MeHg}$ exposure and the development of neurodegenerative disorders, in in vitro studies it has been found that $\mathrm{MeHg}$ can induce Parkinson's-like neurotoxicity similar to 1-methyl-4- phenylpyridinium (Shao et al., 2015), and in vivo it hastens the onset of amyotrophic lateral sclerosis-like phenotype in G93A mice (Johnson et al., 2011). Several reports have suggested that HDACs may play a role in neurodegenerative disease and might be involved in the neurotoxic effects of environmental pollutants. In particular, among the zincdependent HDAC family members, composed of class I (HDACs 1, 2, 3, and 8), classes IIa and IIb (HDACs 4, 5, 6, 7, 9, and 10 ), or class IV (HDAC 11), that are all expressed in the brain (Volmar and Wahlestedt, 2015), only the isoforms 1-6 seem to be involved in neurotoxicity. In fact, it has been demonstrated that HDACs 1, 2, and 4 exert a neurotoxic effect after brain ischemia (Formisano et al., 2015b; Yuan et al., 2016), HDAC3 and HDAC5 can induce cell death of cerebellar granule neurons (CGN) (Bardai and D'Mello, 2011), and HDAC6 inhibition protects against oxidative stress-induced neurodegeneration (Rivieccio et al., 2009). On the other hand a pan HDAC inhibition has been found to be neuroprotective in vitro against the neurotoxicity of bis (2-ethylhexyl) phthalate (DEHP) (Guida et al., 2014), Polychlorinated Biphenyls (PCB) (Formisano et al., 2011, 2015a), and $\mathrm{MeHg}$ (Guida et al., 2015b). Recently, it has also been shown that the mercury-containing organic compound Thimerosal induces an increase of the HDAC4 isoform (Guida et al., 2015b, 2016) determining neuronal cell death, but the molecular mechanism of this effect is still unrevealed. On the other hand, it is known that HDAC4 mRNA expression is regulated by the transcription factors specificity proteins 1 (Sp1) and 3 (Sp3) (Liu et al., 2006), that have been also associated in vivo to neuronal cell death after stroke (Formisano et al., 2015b), and in vitro to cell death after PCB exposure, through the up-regulation of RE1-Silencing Transcription factor (REST) (Formisano et al., 2015c). These findings prompted us to investigate whether HDAC4 is the only isoform involved in MeHg-induced neuronal cell death and the molecular mechanisms responsible for its increase. Furthermore, since it has been demonstrated that male mice offspring of mothers chronically exposed to $\mathrm{MeHg}$ show persistent behavioral changes, associated to lower expression of brain-derived neurotrophic factor (BDNF) mRNA in the hippocampal dentate gyrus (DG) (Ceccatelli et al., 2013), and that $\mathrm{MeHg}$ exposure causes a concentration dependent decrease of serum BDNF in girls born from nonsmoking mothers (Spulber et al., 2010), we investigated whether among the HDAC4 target genes, the BDNF could be involved in neuronal cell death evoked by MeHg. At last in the present study we found that $\mathrm{MeHg}$ increases specifically HDAC4 expression in SH-SY5Y nuroblastoma cells and in rat cortical neurons and its toxic effect is due to the triggering of the $\mathrm{p} 38 / \mathrm{Sp} 1-\mathrm{Sp} 4 / \mathrm{HDAC} 4 / \mathrm{BDNF}$ pathway.

\section{MATERIALS AND METHODS}

\section{Drug and Chemicals}

Methylmercury (II) chloride (MeHg) (cod: 442534 stock solution $100 \mu \mathrm{M}$ ) and p38 inhibitor SB239063 (SB) (cod: 559404 stock solution $10 \mathrm{mM}$ ) were both dissolved in vehicle as previously reported (Sirabella et al., 2012; Guida et al., 2015b). Culture media and sera were purchased from Invitrogen (Milan, Italy). In all experiments, the control group (CTL) was treated with vehicle.

\section{Cell Cultures and Small Interfering RNAs (siRNAs) and Constructs Transfections}

SH-SY5Y human neuroblastoma cells and rat cortical neurons (DIV 7) were cultured as previously reported (Formisano et al., 2013). Importantly, to avoid glial contamination the glial inhibitor cytosine arabinoside $(2.5 \mu \mathrm{M})$ was added at the second day of seeding (Formisano et al., 2013). The experiments on primary cortical neurons were performed according to the experimental protocols approved by the Ethics Committee of "Federico II" University of Naples. SH-SY5Y cells were incubated in a low serum medium (Guida et al., 2015b) and treated with $\mathrm{MeHg} 1 \mu \mathrm{M}$ at 12 and $24 \mathrm{~h}$. For dose-dependent experiments, primary cortical neurons were treated for $24 \mathrm{~h}$ with $\mathrm{MeHg}$ at $0.1,0.5,1$, and $3 \mu \mathrm{M}$. For SB239063 experiments cells were pre-treated for $2 \mathrm{~h}$ with the drug at $10 \mu \mathrm{M}$ followed with $\mathrm{MeHg} 1 \mu \mathrm{M}$ for $24 \mathrm{~h}$. All transfection experiments in $\mathrm{SH}-$ SY5Y and cortical neurons were performed $24 \mathrm{~h}$ before $\mathrm{MeHg}$ treatment $(1 \mu \mathrm{M} / 24 \mathrm{~h})$ with HiPerFect Transfection Reagent (Quiagen) in accordance with the manufacture's protocol, as previously reported (Vinciguerra et al., 2014). In SH-SY5Y cells and cortical neurons the synthetic small interfering RNA (siRNA) transfections for Sp1 (siSp1), HDAC4 (siHDAC4), and negative control (siCTL) were performed as already reported (Formisano et al., 2015b; Guida et al., 2016). In particular, siRNAs against Sp1 reduced Sp1 protein expression respectively of 80 and $68 \%$ in SH-SY5Y cells and rat cortical neurons, as already published (Formisano et al., 2015b). Whereas, siRNAs against HDAC4 reduced HDAC4 protein expression respectively of 75 and $71 \%$ in SH-SY5Y cells and rat cortical neurons, as already published (Guida et al., 2016). They were also transfected with siRNAs against human and rat Sp4 (siSp4; SI04333588) at $20 \mathrm{nM}$, both purchased from Quiagen. For BDNF overexpression cortical neurons were transfected with $15 \mu \mathrm{g}$ of plasmid pCMV6 BDNF (BDNF), that was a gift from Yves-Alain Barde (Addgene plasmid \#39857, (Hofer et al., 1990), or with the empty vector pCMV6 (mock), using Lipofectamine in Optimem for $2 \mathrm{~h} 24 \mathrm{~h}$ before $\mathrm{MeHg}$ treatment $(1 \mu \mathrm{M} / 24 \mathrm{~h})$. The number of cells and the efficiency of transfection was the same as reported in other studies (Formisano et al., 2015b).

\section{Determination of Cell Vitality}

Neuronal vitality was evaluated by measuring lactate dehydrogenase (LDH) efflux into the medium after cortical neurons have been treated with $\mathrm{MeHg}$ for $24 \mathrm{~h}$ at concentrations of $0.1,0.5,1$, and $3 \mu \mathrm{M}$. LDH was determined with LDH Cytotoxicity Kitfrom Cayman, DBA (Milan, Italy), as previously reported (Formisano et al., 2015b). Cell lysate, prepared with 1\% 
Triton X-100 (Sigma-Aldrich), was used as a positive control, and its value was considered $100 \%$.

For the experiments with siRNAs (sip38, siSp1, siSp4, and siHDAC4), neurons were transfected with siRNAs $24 \mathrm{~h}$ before $\mathrm{MeHg}$ treatment $(1 \mu \mathrm{M} / 24 \mathrm{~h})$. Cell viability was measured by MTT assay as previously described (Guida et al., 2015a, 2016).

\section{Chromatin Immunoprecipitation (Chlp) Assay and Quantitative Reverse Transcription Polymerase Chain Reaction (qRT-PCR) Analysis}

ChIP assay in SH-SY5Y cells was performed as previously reported (Guida et al., 2015a). The antibodies for immunoprecipitation are: anti-Sp1 (sc- 14027 Santa Cruz Biotechnology), anti-Sp4 (sc- 645 Santa Cruz Biotechnology), and anti-HDAC4 (sc- 11418 Santa Cruz Biotechnology), all used at $3 \mu \mathrm{g}$. The specificity of antibodies used for ChIP assays has been confirmed by our group and others (Saramäki et al., 2009; de Nigris et al., 2010; Johar et al., 2013; Formisano et al., 2015b) where it has been found with this technique that Sp1, Sp4, and HDAC4 are bound to the promoter sequences of their target genes. Furthermore, since the antibodies used were all rabbit polyclonal, in all the experiments Rabbit IgG antibody (sc-2345 Santa Cruz Biotechnology) was used as a negative control (data not shown). Two-microliter aliquots (in duplicate for each treatment group) were used for PCR with Fast SYBR green master mix (Applied Biosystems). Data obtained from three different PCR experiments were expressed as a percentage of the control, all normalized for the DNA input. The oligonucleotides used for the amplification of immunoprecipitated DNA of HDAC4 promoter and BDNF promoter IV sequences were already published (Liu et al., 2006; Sen et al., 2015). For qRT-PCR analysis, endogenous RNA (2 $\mu \mathrm{g}$ ) from SH-SY5Y was isolated according to the supplier's protocol (Tri reagent; Sigma-Aldrich) and treated with DNAseI $(1 \mathrm{U} / \mu \mathrm{l})$ for $15 \mathrm{~min}$ at room temperature. Retrotranscription and q-PCR reaction were performed as already published (Formisano et al., 2015c). Primer pairs for human HDAC4, L19 ribosomal protein (L19) and BDNF splicing isoform IV were already published (Guida et al., 2014; Sen et al., 2015). Three independent samples were amplified simultaneously in triplicate in one assay run, and the threshold cycle $(\mathrm{Ct})$ value for each experimental group was determined. L19 was used for normalization and differences among groups was evaluated with the $2^{-\Delta \Delta \text { ct }}$ formula.

\section{Immunoblotting}

Cell lysates from cortical neurons and SH-SY5Y cells were prepared via scraping on ice and pelleting at $4^{\circ} \mathrm{C}$, followed by resuspension in lysis buffer $(50 \mathrm{mM}$ Tris- $\mathrm{HCl}(\mathrm{pH} 7.5)$, $150 \mathrm{mM} \mathrm{NaCl}, 1 \% \mathrm{NP}-40,1 \mathrm{mM}$ EDTA, $10 \mathrm{mM}$ sodium pyrophosphate, $1 \mathrm{mM}$ sodium orthovanadate, $100 \mathrm{mM} \mathrm{NaF}$, $1 \mathrm{mM}$ PMSF and protease inhibitor cocktail), and kept in ice for $1 \mathrm{~h}$. After that cells were centrifuge at 14,000 rpm and protein concentration in the supernatant was quantified by Bradford method and resuspended in lamely buffer. For immunoblotting, $100 \mu \mathrm{g}$ of sample for BDNF and $50 \mu \mathrm{g}$ of sample for all other proteins were separated via SDS-PAGE and transferred to PVDF membranes. After transfer, the membranes were blocked for $2 \mathrm{~h}$ at room temperature with 5\% non-fat milk in phosphate-buffered saline (PBS) and then incubated at the dilution of 1:1000 in 5\% non-fat milk with the following primary antibodies: anti-Sp1, anti-Sp3, anti-Sp4, anti P-ERK, anti-ERK-, anti P-p38, anti-p38, anti P-JNK, anti-JNK, antiHDAC1, anti-HDAC2, anti-HDAC3, anti-caspase 3, anti- $\alpha$ tubulin and anti- $\beta$-actin, that were already used (Formisano et al., 2015a,b; Guida et al., 2015b), anti-HDAC4 (cod. sc-11418, 1:1000 polyclonal rabbit antibody; Santa Cruz Biotechnology), anti-HDAC5 (cod. sc-11419, 1:1000 polyclonal rabbit antibody; Santa Cruz Biotechnology), anti-HDAC6 (cod. sc-11491, 1:1000 polyclonal goat antibody; Santa Cruz Biotechnology). $\beta$-actin or $\alpha$-tubulin were used as the loading control for all the experiments. After that the membrane were washed with PBS with $0.1 \%$ Tween, and finally were probed with secondary antibodies. The membranes were exposed to film after enhanced chemiluminescence (ECL) (Amersham Biosciences, Piscataway, NJ). Quantitative analysis of immunoblots was performed from images with unsaturated conditions and quantized with Image J software.

\section{Statistical Analysis}

For the analysis of more than two experimental groups was used ANOVA followed by Turkey's test as the post-hoc test, whereas for the analysis of two experimental groups was used Student's $t$-test. Details of the statistical analyses are described in the figure legends.

\section{RESULTS}

\section{MeHg Up-Regulates HDAC4 mRNA and Protein}

Since it has been found in SH-SY5Y cells that HDAC-inhibition reverts the neurodetrimental effect of $\mathrm{MeHg}$ at $1 \mu \mathrm{M} / 24 \mathrm{~h}$ (Guida et al., 2015b), we evaluated the protein levels of the neurotoxic HDAC isoforms 1-6 (Rivieccio et al., 2009; Bardai and D'Mello, 2011; Formisano et al., 2015b; Yuan et al., 2016) performing Western blot after treating cells with $\mathrm{MeHg}$ for $24 \mathrm{~h}$. $\mathrm{MeHg}(1 \mu \mathrm{M})$ significantly increased HDAC4, but not HDACs 1,2,3, and 5,6 (Figures 1A-F). In addition, qRT-PCR analysis revealed that $\mathrm{MeHg}$-induced HDAC4 increase occurred also at transcriptional level at 12 and $24 \mathrm{~h}$ (Figure 1G).

\section{MeHg-Induced HDAC4 mRNA and Protein Increase Is Determined by Sp1 and Sp4 Up-Regulation}

Since HDAC4 expression can be regulated by $\mathrm{Sp}$ family transcription factors (Liu et al., 2006), we evaluated the effect of $\mathrm{MeHg}$ exposure on the expression of Sp1, Sp3, and Sp4 in SHSY5Y cells. In these cells at 12 and $24 \mathrm{~h} \mathrm{MeHg}(1 \mu \mathrm{M})$ induced an increase in $\mathrm{Sp} 1$ and $\mathrm{Sp} 4$, but not Sp3 protein expression (Figures 2A-C). Importantly, as revealed by ChIP experiments, Sp1 and Sp4 increase occurred also at level of HDAC4 gene promoter sequence (Figures 2D,E). To evaluate the effect of Sp 
A

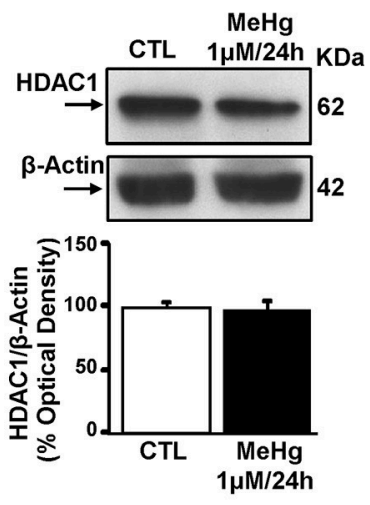

E
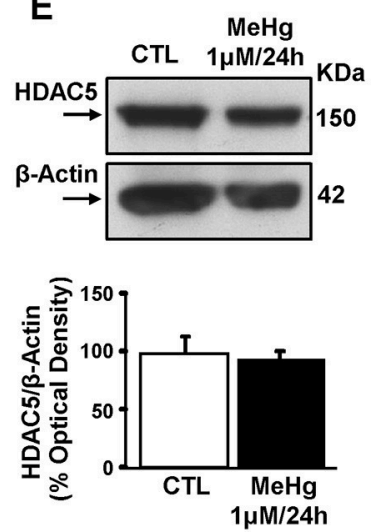

B
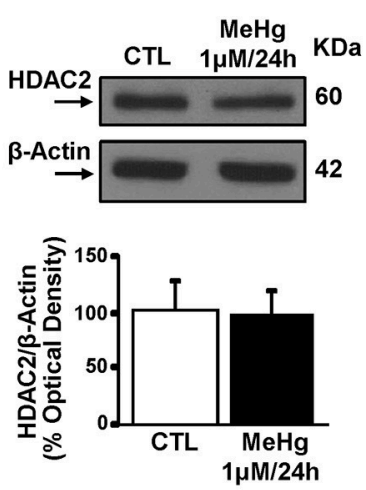

$\mathbf{F}$
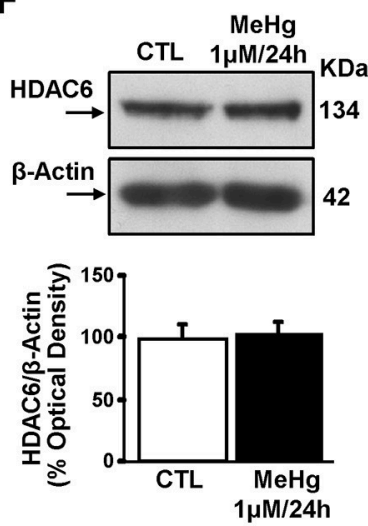

C

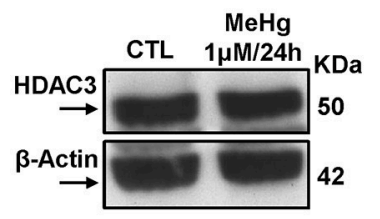

D
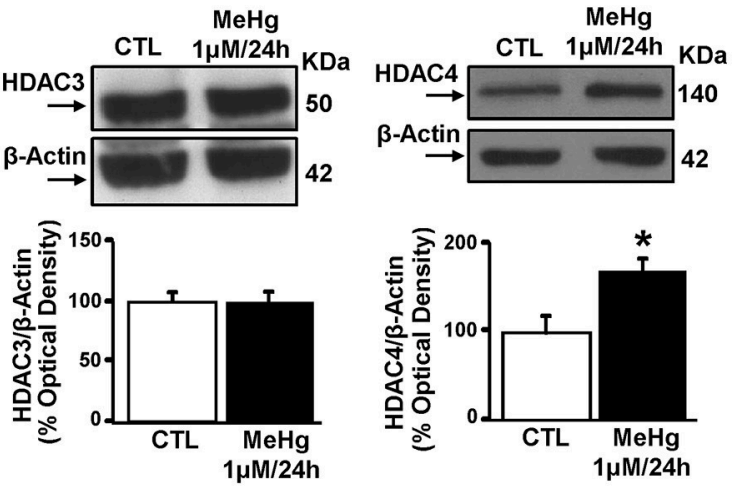

$\mathbf{G}$

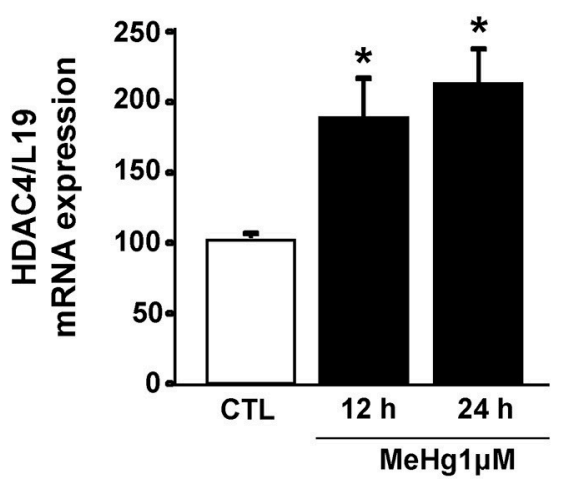

FIGURE $\mathbf{1}$ | Effect of $\mathbf{2 4} \mathbf{h}$ of Methylmercury (1 $\boldsymbol{\mu} \mathbf{M})$ on HDACs protein expression. (A-F) Western blots of HDAC1, HDAC2, HDAC3, HDAC4, HDAC5, and HDAC6 in SH-SY5Y cells treated for $24 \mathrm{~h}$ with $\mathrm{MeHg}(1 \mu \mathrm{M})$. Bars represent the mean \pm S.E.M. obtained from three independent experiments. Asterisk symbols on columns indicate differences between control (CTL) and MeHg. ${ }^{*} p \leq 0.05$ (unpaired $t$-test). (G) qRT-PCR of HDAC4 in SH-SY5Y treated for 12 or 24 h with MeHg $(1 \mu \mathrm{M})$. Graphs show quantification of ratio of HDAC4 to L19. Bars represent mean \pm S.E.M. obtained from three independent experiments. Asterisk symbols on columns indicate differences between CTL and MeHg treatment. ${ }^{*} P \leq 0.05$ (one-way ANOVA with Turkey's post-hoc test).

transcription factors on HDAC4 mRNA and protein expression levels after MeHg treatment, SH-SY5Y cells were silenced with siRNAs against Sp1 (siSp1) and Sp4 (siSp4). The effect of siSp1 on Sp1 protein expression it has been already published (Formisano et al., 2015c), whereas siSp4 reduced Sp4 protein expression by $60 \%$ compared to cells transfected with siCTL (data not shown). Interestingly, as shown in Figures 2F,G, siSp1 and siSp4 significantly blocked $\mathrm{MeHg}$-induced HDAC4 mRNA and protein increase.

\section{MeHg-Induced HDAC4 mRNA and Protein Up-Regulation Is due to an Increase of p38 Phosphorylation That Increases Sp1 and Sp4 Binding on HDAC4 Promoter Sequence}

As $\mathrm{MeHg}$ induces the phosphorylation of ERK1/2 and p38, but not of JNK (Lu et al., 2011), we investigated whether MeHginduced Sp1 and Sp4 up-regulation occurred via the activation of a specific MAP Kinase. In these cells, $\mathrm{MeHg}(1 \mu \mathrm{M})$ at 12 and $24 \mathrm{~h}$ caused an increase of p38 phosphorylation, but not of ERK 1/2 and JNK (Figures 3A-C). Interestingly, in cells exposed to $\mathrm{MeHg}$, pre-treatment with the p38 inhibitor SB239063 significantly counteracted the increase of Sp1 and Sp4 protein expression (Figures 3D,E). To evaluate whether the increase in p38 phosphorylation following $\mathrm{MeHg}$ exposure could contribute to increases in HDAC4 mRNA and protein expression through Sp1 and Sp4, we performed a ChIP assays on HDAC4 promoter sequence for Sp1 and Sp4, and qRT-PCR and Western blotting experiments to analyze the modifications of HDAC4 mRNA and protein. As shown in Figures 3F-H, SB239063 treatment reduced $\mathrm{MeHg}$-induced binding of Sp1 and Sp4 to the HDAC4 gene promoter sequence with consequent reduction of $\mathrm{MeHg}$ increased HDAC4 mRNA and protein expression.

\section{p38/Sp1/Sp4 Increase the Binding of HDAC4 on BDNF Promoter IV Sequence Determining Its mRNA and Protein \\ Reduction}

Given that the HDAC4 gene target BDNF expression has been correlated to $\mathrm{MeHg}$ exposure (Spulber et al., 2010; Ceccatelli 

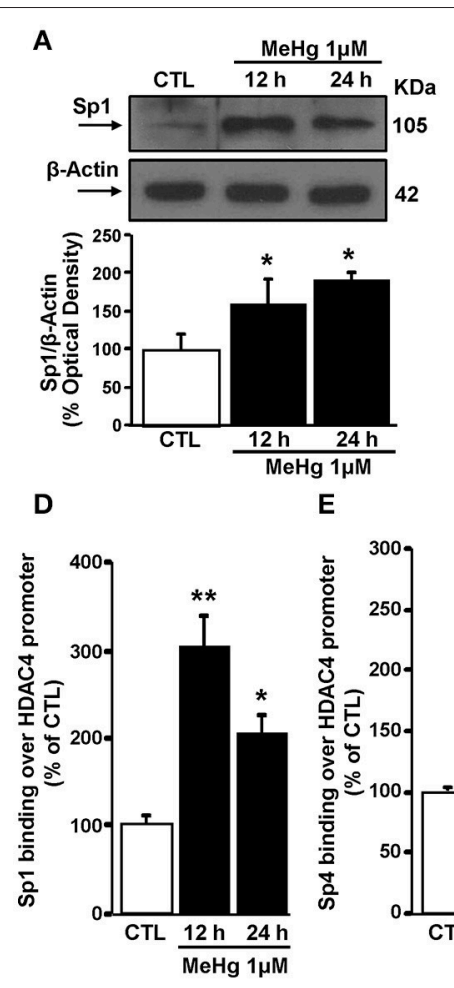

E
B
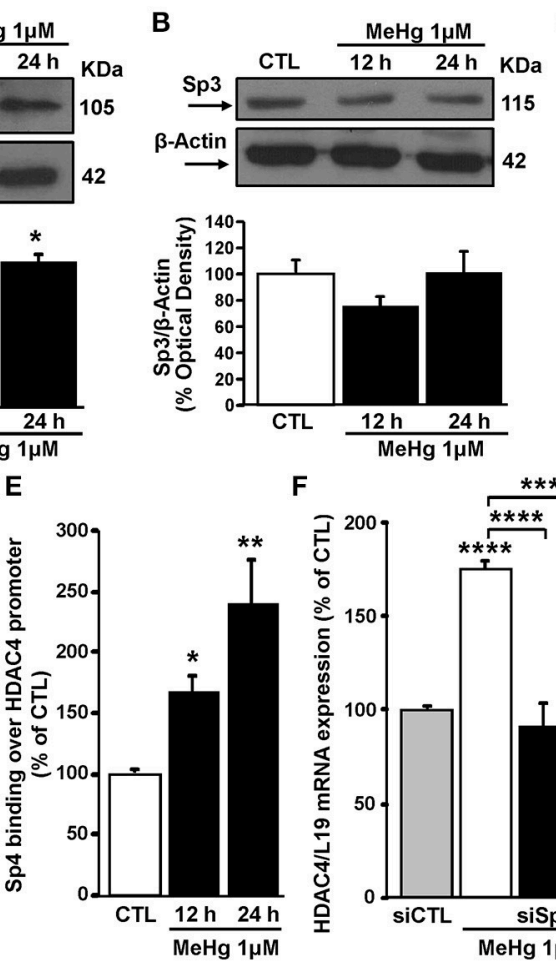

$\mathbf{F}$

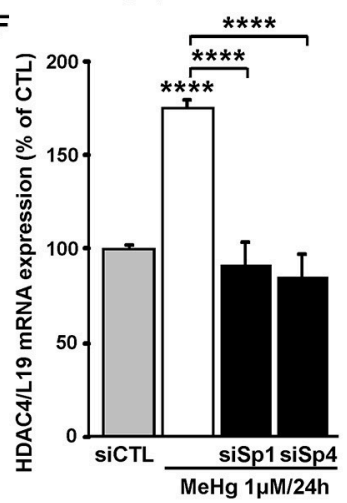

C

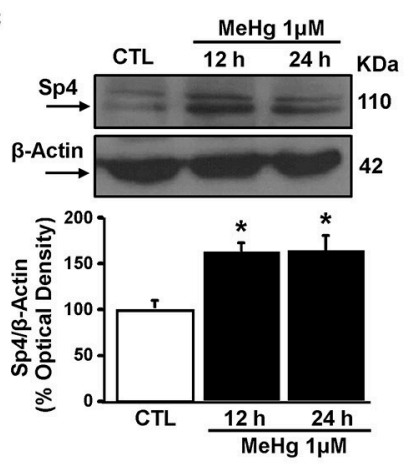

G

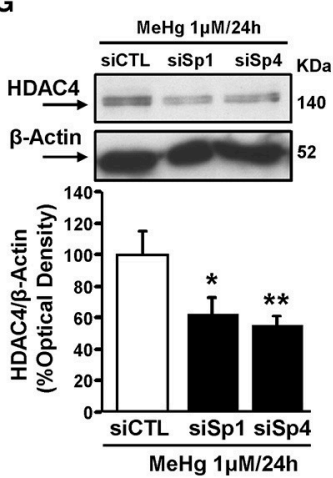

FIGURE 2 | Sp1 and Sp4 increase and bind HDAC4 gene promoter sequence, determining its up-regulation in SH-SY5Y treated with MeHg (1 $\mu$ M). (A-C) Western blotting of Sp1, Sp3, and Sp4 in SH-SY5Y cells treated with $1 \mu \mathrm{M}$ of MeHg for 12 and $24 \mathrm{~h}$. Bars represent the mean \pm S.E.M. obtained from three independent experiments. Asterisk symbols on columns indicate differences between CTL and MeHg treatment. ${ }^{*} p<0.05$ (one-way ANOVA with Turkey's post-hoc test). (D,E) ChIP analysis of the Sp1 sites on HDAC4 gene promoter regions carried out with anti-Sp1 and anti-Sp4, in SH-SY5Y cells treated with $1 \mu \mathrm{M}$ of MeHg for 12 and $24 \mathrm{~h}$. The binding activity of Sp1 and Sp4 is graphically represented as the percentage of the control (CTL). Each column represents the mean \pm S.E.M $(n=3)$. Asterisk symbols on columns indicate differences between CTL and MeHg treatment. ${ }^{\star *} p<0.01,{ }^{\star} p<0.05$ (one-way ANOVA with Turkey's post-hoc test). (F) qRT-PCR of HDAC4 in SH-SY5Y transfected with siRNA for Sp1 (siSp1) and for Sp4 (siSp4) and treated with MeHg (1 $\mu$ M/24 h). Graphs show quantification of ratio of HDAC4 to L19. Bars represent mean \pm S.E.M. obtained from three independent experiments. Asterisk symbols on columns indicate differences between siCTL and MeHg. Asterisk symbols on brackets indicate significance compared to MeHg. ${ }^{\star \star \star \star} p<0.0001$ (one-way ANOVA with Turkey's post-hoc test). (G) Western blotting of HDAC4 in SH-SY5Y transfected with siRNA for Sp1 (siSp1) and for Sp4 (siSp4) and treated with MeHg (1 $\mu \mathrm{M} / 24 \mathrm{~h})$. Bars represent the mean \pm S.E.M. obtained from three independent experiments. Asterisk symbols on columns indicate differences compared to siCTL+MeHg. ${ }^{*} p<0.01,{ }^{*} p<0.05$ (one-way ANOVA with Turkey's post-hoc test).

et al., 2013), that in SH-SY5Y cells the BDNF exon IV and VI splicing isoforms represent the most highly expressed transcripts (Donnici et al., 2008), and that only BDNF promoter IV (BDNF pIV) activity is repressed by HDAC4 (Koppel and Timmusk, 2013), and we investigated the effect of $\mathrm{MeHg}$ at $24 \mathrm{~h}$ on the BDNF exon IV expression. As shown in Figures 4A,B MeHg reduced BDNF exon IV expression, in parallel with an increase of HDAC4 binding on BDNF promoter IV sequence. Furthermore, we studied the role of $\mathrm{p} 38 / \mathrm{Sp} 1 / \mathrm{Sp} 4 / \mathrm{HDAC} 4$ pathway in the modulation of HDAC4 binding on BDNF pIV sequence and on BDNF IV mRNA expression. Interestingly, as shown in Figure 4C, $\mathrm{MeHg}$ determined an increase of HDAC4 binding on BDNF pIV, that was counteracted when cells were pretreated with SB239063, or transfected with siRNAs against Sp1, Sp4, and HDAC4. As expected, we found that MeHg-induced reduction of BDNF mRNA was blocked when cells were pretreated with SB239063, or transfected with siRNAs against Sp1, Sp4, and HDAC4 (Figure 4D).

\section{MeHg Exposure Induces Cell Death by Activation of $\mathrm{p} 38 / \mathrm{Sp} 1 / \mathrm{Sp} 4 / \mathrm{HDAC} 4 / \mathrm{BDNF}$ Axis in Cortical Neurons}

Cortical neurons were exposed to $\mathrm{MeHg}(0.1-3 \mu \mathrm{M}$; Figure 5A) for $24 \mathrm{~h}$. As shown in Figure 5A a dose-related increase in cell death was observed, as evidenced by LDH assay, and $1 \mu \mathrm{M}$ was the concentration of $\mathrm{MeHg}$ that determined cell death by about $50 \%$ and was therefore chosen for all the experiments in cortical neurons. Notably, $\mathrm{MeHg}$ induced an increase of P-p38, Sp1, Sp4, HDAC4 in parallel with a reduction of BDNF protein (Figures 5B-F). Then we studied the role of P-p38/Sp1/Sp4/HDAC4/BDNF pathway in MeHginduced cell death. To this aim, we knocked down p38, Sp1, Sp4 and HDAC4, by transfecting neurons with specific siRNAs named sip38, siSp1, siSp4, and siHDAC4 and increased BDNF with a plasmid overexpressing BDNF named pBDNF. Notably, the efficiency of sip38, siSp1, and siHDAC4 to reduce respectively $\mathrm{p} 38, \mathrm{Sp} 1$, and HDAC4 protein expression 


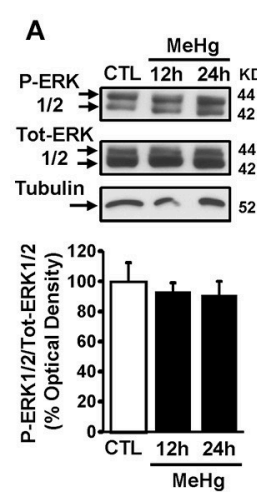

$\mathbf{F}$



B $\frac{\mathrm{MeHg}}{12 \mathrm{~h} 24 h}$

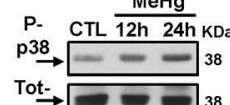

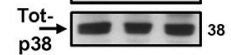

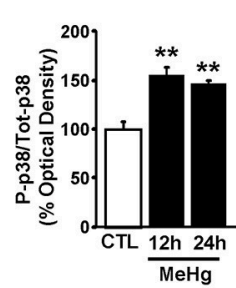

G

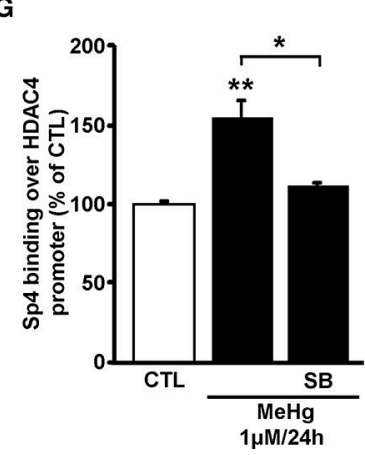

C
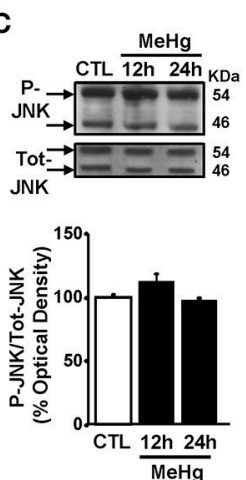

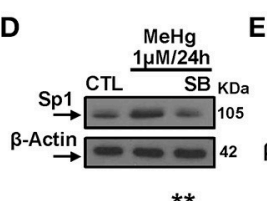

E
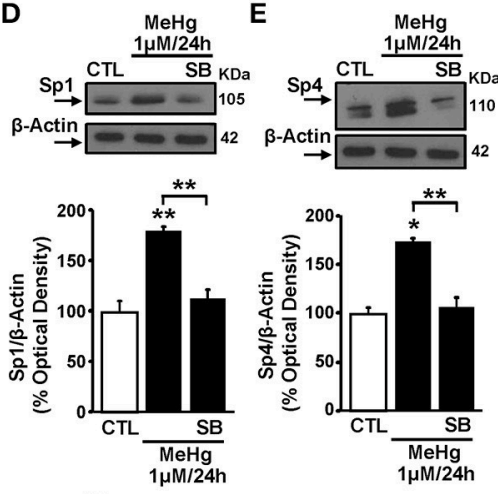

H

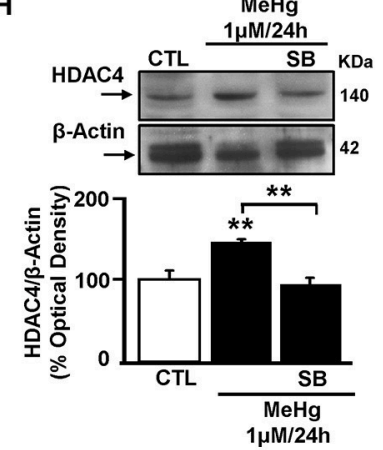

FIGURE 3 | p38 MAPK inhibitor SB239063 reverts Sp1 and Sp4 protein expression increase and blocks their binding on HDAC4 promoter sequence and its down-regulation. (A-C) Western blotting of P-ERK1/2, P-p38, and P-JNK in SH-SY5Y cells treated with $1 \mu \mathrm{M}$ of MeHg for 12 and 24 h. Bars represent the mean \pm S.E.M. obtained from three independent experiments. Asterisk symbols on columns indicate differences between CTL and MeHg. ${ }^{\star \star} p<0.01$ (one-way ANOVA with Turkey's post-hoc test). (D,E) Western blotting of Sp1 and Sp4 in SH-SY5Y cells treated with MeHg (1 $\mu$ M/24 h), alone or in combination with SB239063 (SB). Bars represent the mean \pm S.E.M. obtained from three independent experiments. Asterisk symbols on columns indicate differences compared to CTL. Asterisk symbols on brackets indicate significance between MeHg and SB. ${ }^{* *} p<0.01,{ }^{*} p<0.05$ (one-way ANOVA with Turkey's post-hoc test). (F,G) ChIP analysis of the Sp1 sites on HDAC4 gene promoter regions carried out with anti-Sp1 and anti-Sp4, in SH-SY5Y cells treated with MeHg (1 $\mu$ M/24 h), alone or in combination with SB. The binding activity of Sp1 and Sp4 is graphically represented as the percentage of the control. Each column represents the mean $\pm \mathrm{S}$.E.M ( $n=3)$. Asterisk symbols on columns indicate differences between control CTL and MeHg. Asterisk symbols on brackets indicate significance between MeHg and SB+MeHg. ${ }^{\star *} p<0.01$, ${ }^{*} p<0.05$ (one-way ANOVA with Turkey's post-hoc test). (H) Western blotting of HDAC4 in SH-SY5Y cells treated with MeHg (1 $\mu$ M/24 h), alone or in combination with SB. Bars represent the mean \pm S.E.M. obtained from three independent experiments. Asterisk symbols on columns indicate differences between CTL and MeHg. Asterisk symbols on brackets indicate significance between MeHg and SB+MeHg. ** $p<0.01$ (one-way ANOVA with Turkey's post-hoc test).

was previously published (Sirabella et al., 2012; Formisano et al., 2015b; Guida et al., 2015b, 2016). Instead, siSp4 significantly reduced Sp4 expression by $65 \%$, whereas pBDNF increased BDNF expression by $54 \%$, both compared to the respective control (Figures $\mathbf{5 G}, \mathbf{H}$ ). Intriguingly, transfection of sip38, siSp1, siSp4, siHDAC4, and pBDNF significantly reduced $\mathrm{MeHg}$-induced cell death, as revealed by MTT assay (Figure 5I).

\section{DISCUSSION}

The results of the present study showed that $\mathrm{MeHg}$ exposure increased the expression of the HDAC4 isoform in SH-SY5Y cells and that the stimulation of the P-p38/Sp1/Sp4 pathway was the mechanism by which HDAC4 mRNA and protein are up-regulated during $\mathrm{MeHg}$ exposure. In addition evidence is provided that HDAC4 reduced the expression of its target gene $\mathrm{BDNF}$ and that in cortical neurons treated for $24 \mathrm{~h}$ with $\mathrm{MeHg}$ the knocking down of p38, Sp1, Sp4, and HDAC4 or the overexpression of BDNF caused a reduction of cell death.

The most interesting result of this work is that HDAC4 is identified as a novel target of $\mathrm{MeHg}$-induced neuronal toxicity. In fact, $\mathrm{MeHg}$ exposure increased HDAC4 gene and protein expression causing also in the nucleus an increase of its binding to the BDNF gene promoter IV sequence with consequent $\mathrm{mRNA}$ and protein reduction of this neuroprotective factor. These results are in accordance with recent in vivo studies demonstrating that intramuscular injection of the organomercury compound thimerosal induces neuronal cell death by increasing HDAC4 expression in rat prefrontal cortex (Guida et al., 2015b, 2016), and that in cortical neurons HDAC4 knockdown improves neuronal survival after oxygen glucose deprivation (OGD, Yuan et al., 2016). Furthermore, we found that of the three main branches of the MAPK cascade (ERK, p38, and JNK), only p38 was involved in regulating HDAC4 gene expression in cells exposed to $\mathrm{MeHg}$. In fact, $\mathrm{MeHg}$ increased p38 phosphorylation, but not that of ERK 

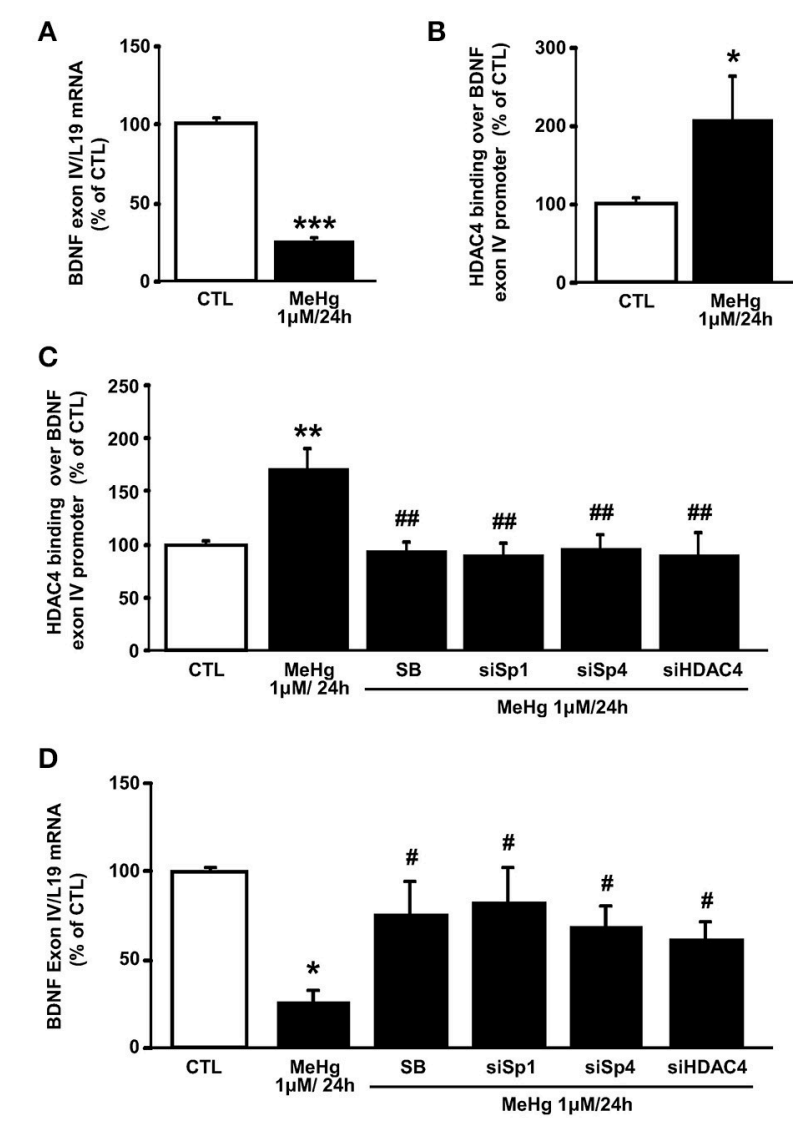

FIGURE 4 | MeHg increases HDAC4 binding on BDNF exon IV promoter, by the $\mathrm{p} 38 / \mathrm{Sp} 1 / \mathrm{Sp} 4$ axis, determining its gene reduction. (A) qRT-PCR of BDNF exon IV in SH-SY5Y treated with MeHg (1 $\mu \mathrm{M} / 24 \mathrm{~h})$. Graphs show quantification of ratio of BDNF exon IV to L19. Bars represent mean \pm S.E.M. obtained from three independent experiments. ${ }^{\star \star \star} p<0.001$ (unpaired $t$-test). (B) ChIP analysis of the BDNF exon IV promoter regions carried out with anti-HDAC4, in SH-SY5Y cells treated with MeHg $(1 \mu \mathrm{M} / 24 \mathrm{~h})$. The binding activity of HDAC4 is graphically represented as the percentage of CTL. Each column represents the mean \pm S.E.M $(n=3)$. ${ }^{*} p<0.05$ (unpaired t-test). (C) ChIP analysis of the BDNF exon IV promoter regions carried out with anti-HDAC4, in SH-SY5Y cells treated with $\mathrm{MeHg}(1 \mu \mathrm{M} / 24 \mathrm{~h})$, alone or in combination with SB SB239063, and treated with $\mathrm{MeHg}$ and transfected with siRNA for Sp1 (siSp1), Sp4 (siSp4), or HDAC4 (siHDAC4). The binding activity of HDAC4 is graphically represented as the percentage of (CTL). Each column represents the mean \pm S.E.M $(n=3)$. Asterisk symbols on columns indicate differences between control CTL and MeHg. Hashtag symbols on columns indicate significance compared to $\mathrm{MeHg} .{ }^{* \star} p<0.01$, ${ }^{\# \#} p<0.01$ (one-way ANOVA with Turkey's post-hoc test). (D) qRT-PCR of BDNF exon IV in $\mathrm{SH}-\mathrm{SY} 5 \mathrm{Y}$ treated with $\mathrm{MeHg}(1 \mu \mathrm{M} / 24 \mathrm{~h})$, alone or in combination with SB, and treated with MeHg and transfected with siRNA for Sp1 (siSp1), Sp4 (siSp4), or HDAC4 (siHDAC4). Graphs show quantification of ratio of BDNF exon IV to L19. Bars represent mean \pm S.E.M. obtained from three independent experiments. Asterisk symbols on columns indicate differences between control CTL and MeHg. Hashtag symbols on columns indicate significance compared to MeHg. ${ }^{*} p<0.05,{ }^{*} p<0.05$ (one-way ANOVA with Turkey's post-hoc test).

and JNK, and increased the expression of HDAC4 gene. This hypothesis is straightened by the findings showing that when cells were pretreated with the p38 inhibitor SB239063, the $\mathrm{MeHg}$-induced overexpression of HDAC4 was prevented. That
$\mathrm{MeHg}$ regulates p38, but not ERK and JNK, is partially in agreement with other studies, where it has been found in Neuro2A cell line that $\mathrm{MeHg}$ induced the phosphorylation of ERK1/2 and p38, but not of JNK (Lu et al., 2011), and that in SH-SY5Y cells p38 phosphorylation was stimulated by $\mathrm{MeHg}$, determining apoptotic cell death (Posser et al., 2010).

The MeHg-induced HDAC4 up-regulation seems to occur through the participation of the transcription factors Sp1 and Sp4, since the expression of these proteins was increased during $\mathrm{MeHg}$ exposure and HDAC4 increase was reverted by silencing of these transcription factors. These findings, together with those showing that the p38 inhibitor was not only able to revert $\mathrm{MeHg}$-induced overexpression of HDAC4 but also that of Sp1 and Sp4, strongly suggest that the pathway P$\mathrm{p} 38 / \mathrm{Sp} 1 / \mathrm{Sp} 4$ is responsible for HDAC4 overexpression. These results are in partial accordance with those obtained by Liu et al. (2006), where they found that Sp1 and Sp3, by binding HDAC4 promoter sequence, up-regulated HDAC4 expression whereas, in our model, Sp4 and not Sp3 was modulated after $\mathrm{MeHg}$ treatment. That $\mathrm{Sp} 4$ isoform, specifically expressed in the brain (Suske, 1999), is regulated by p38 and its increase up-regulates HDAC4 gene and protein expression after $\mathrm{MeHg}$ exposure, to our knowledge, appears to be a novel evidence.

The activation of HDAC4 triggered by $\mathrm{MeHg}$ is associated with the down-regulation of its target gene BDNF and these in vitro results are in line with those in vivo obtained by Ceccatelli et al. (2013) that found a decrease of BDNF content in the hippocampal DG of male mice born from mothers exposed to $\mathrm{MeHg}$. These results appear rather interesting since, at molecular level, BDNF human and rat genes are very similar. In particular BDNF gene is composed of eight 5-non-coding exons and one 3-coding exon. Individual promoters control the expression of each of the eight 5-exons, that splice with the common exon 3 encoding for BDNF protein ( $\mathrm{He}$ et al., 2010). BDNF IV and VI exons are the most highly expressed transcripts in humans, representing over $80 \%$ of the total BDNF (Donnici et al., 2008). Interestingly, BDNF promoter IV is known for its role in mediating activity-dependent BDNF transcription (Tao et al., 1998) and it has been reported that HDAC4 and HDAC5 repressed BDNF promoter IV activity in cortical neurons (Koppel and Timmusk, 2013). It should be underlined that in the present study, in line with the findings of Koppel and Timmusk, we found an increase of HDAC4 binding on the BDNF human promoter sequence IV in parallel with a reduction of $\mathrm{BDNF}$ at level of the splicing isoform IV. The role of HDAC4 in modulating the expression of BDNF following $\mathrm{MeHg}$ exposure is confirmed by the data showing that siRNA against HDAC4 blocked MeHg-induced BDNF gene and protein decrease.

In cortical neurons we obtained results in line with those obtained in SH-SY5Y cells and demonstrated that the pathway p38/Sp1-Sp4/HDAC4/BDNF is responsible for MeHg-induced neuronal death. In fact we found that this pathway is activated after $\mathrm{MeHg}$ treatment (1 $\mathrm{MM} 24 \mathrm{~h}$ ) and that p38, Sp1, Sp4, and HDAC4 silencing or BDNF overexpression partially reverted 

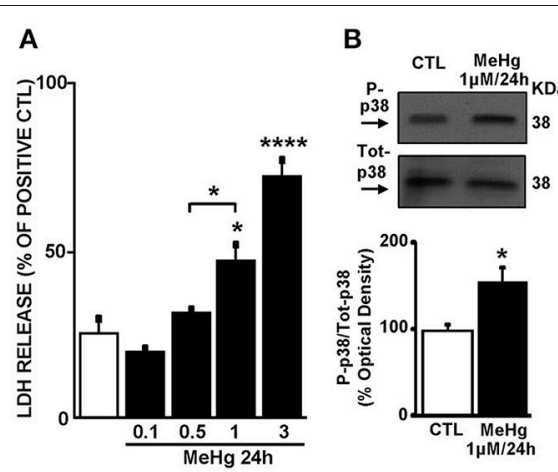

C
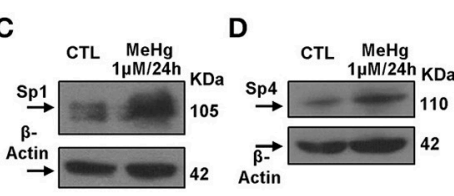

E
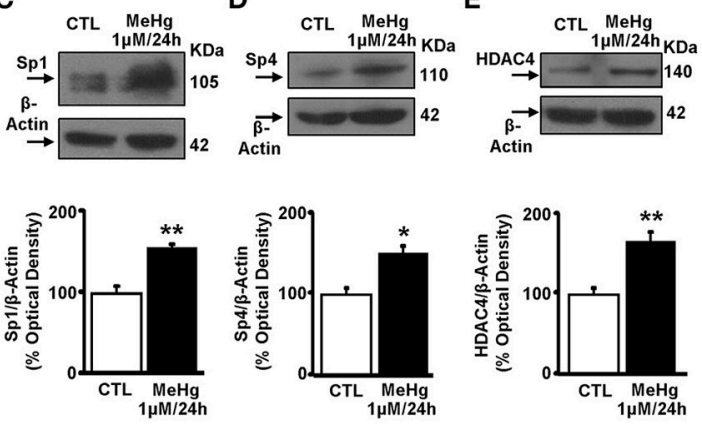

I

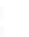

F

G

H
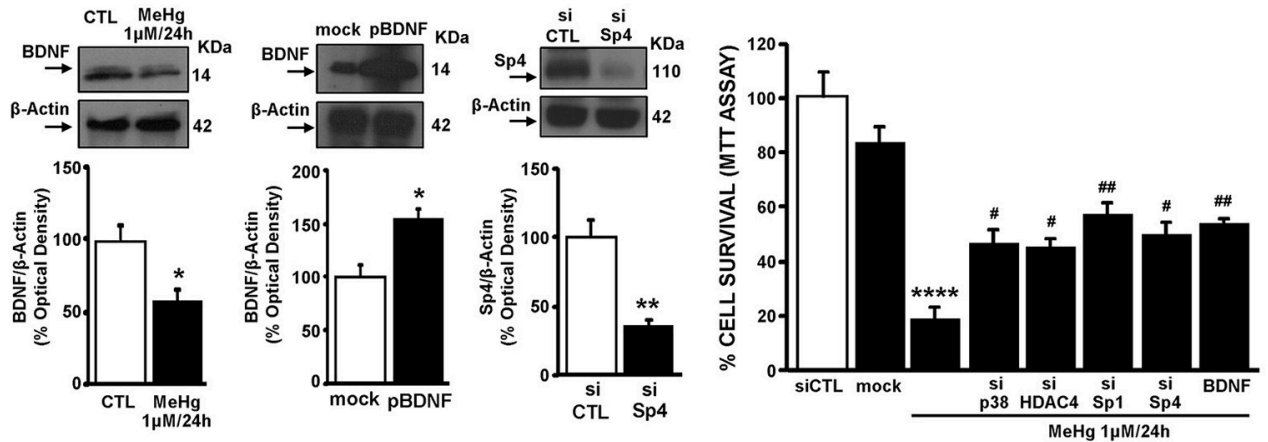

FIGURE 5 | MeHg by activating the p38/Sp1/Sp4/BDNF axis induced cell death in cortical neurons. (A) Effect of $24 \mathrm{~h}$ of MeHg (0.1, $0.5,1$, and $3 \mu \mathrm{M})$ exposure on $\mathrm{LDH}$ release in cortical neurons. Bars represent the mean \pm S.E.M. obtained in three independent experiments. Asterisk symbols on columns indicate differences between CTL and MeHg treatment. Asterisk symbols on brackets indicate differences between MeHg groups. ${ }^{\star} p<0.05$, ${ }^{\star \star * \star} p<0.0001$ (one-way ANOVA with Turkey's post-hoc test). (B-F) Western blotting of P-p38, Sp1, Sp4, HDAC4, and BDNF in cortical neurons treated with $1 \mu$ M of MeHg for 24 h. Bars represent the mean \pm S.E.M. obtained from three independent experiments. Asterisk symbols on columns indicate differences between $\mathrm{CTL}$ and $\mathrm{MeHg}$. ${ }^{* *} p<0.01,{ }^{*} p<0.05$ (unpaired $t$-test). (G) Western blotting of BDNF in cortical neurons transfected for $24 \mathrm{~h}$ with plasmid overexpressing BDNF or with the empty vector (mock). Bars represent the mean \pm S.E.M. obtained from three independent experiments. ${ }^{\star} p<0.05$ (unpaired $t$-test). (H) Western blotting of Sp4 in cortical neurons transfected for $24 \mathrm{~h}$ with a specific siRNA for Sp4 or with scramble (siCTL). Bars represent the mean \pm S.E.M. obtained from three independent experiments. ${ }^{\star *} p<0.01$ (unpaired $t$-test). (I) Effect of $24 \mathrm{~h}$ of $\mathrm{MeHg}(1 \mu \mathrm{M})$ exposure on LDH release in cortical neurons transfected with siRNAs for p38, Sp1, Sp4, and HDAC4 or with pBDNF. Bars represent the mean \pm S.E.M. obtained in three independent experiments. Asterisk symbols on columns indicate differences between CTL and MeHg treatment. Hashtag symbols on columns indicate significance compared to MeHg. \#\# $p<0.01$, \# $p<0.05$ (one-way ANOVA with Turkey's post-hoc test).

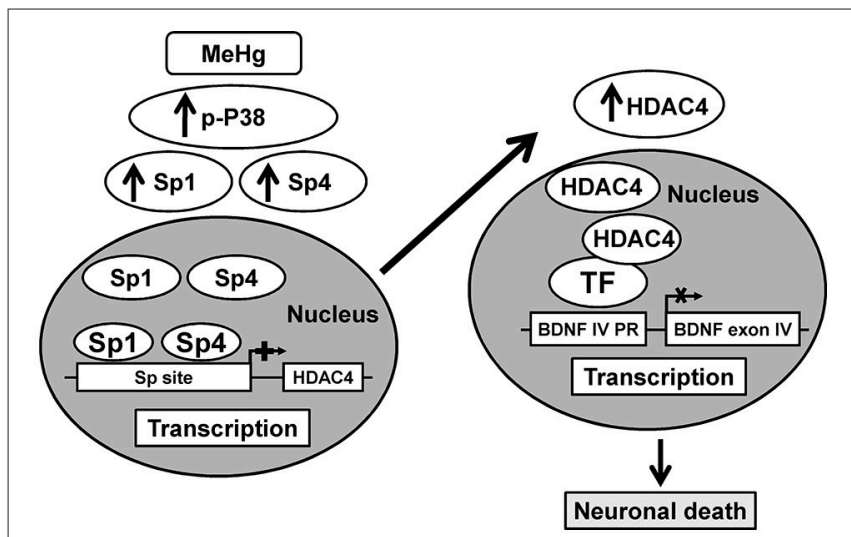

FIGURE 6 | Schematic illustration of p38/Sp1/Sp4/HDAC4/BDNF pathway activated by $\mathrm{MeHg}$ in cortical neurons.

MeHg-induced cell death, since $\sim 20 \%$ of the cells were rescued and $50 \%$ remained dead, thus indicating that other pathways could be involved.
It is noteworthy that in this study we evaluated $\mathrm{MeHg}$ toxicity only in neurons, since glial inhibitor was added to the incubation medium, in order to exclude a participation of glial cells to $\mathrm{MeHg}$ toxic effect. In fact, it has been reported that $\mathrm{MeHg}$ through glial cells modulates neurotoxicity by inhibiting astrocytic glutamate uptake, and stimulating glutamate efflux that causes an increase of glutamate in the synapse and, consequently cell death (Ni et al., 2011). The concentration of MeHg used for the experiments in cortical neurons was the same used in neuroblastoma cells experiments and also in other studies (Fujimura and Usuki, 2012). The neurotoxic role of p38, Sp1, and HDAC4 and the neuroprotective role of BDNF is already known. Indeed: (1) stimulation of the p38 signaling pathways contributes to neuronal cell death (Xia et al., 1995); (2) Sp1 knockdown improves survival in cellular and animal models of Huntington's disease (Qiu et al., 2006) or after PCB exposure (Formisano et al., 2015c); (3) inactivation of HDAC4 by small interfering RNA reduced neuronal cell death to potassium withdrawal (Bolger and Yao, 2005) or after OGD (Yuan et al., 2016); (4) BDNF treatment concurrent with $A \beta 1-42$ exposure prevented neuron death (Nagahara et al., 2009). However, the results of 
the present study may represent a novelty in identifying for the first time the neurotoxicity of Sp4 isoform, as demonstrated by the fact that its knocking-down in cortical neurons reduced $\mathrm{MeHg}$-induced cell death and in proving the involvement of p38/Sp1/Sp4/HDAC4/BDNF chain in eliciting the neurotoxic effects of $\mathrm{MeHg}$ (Figure 6).

\section{AUTHOR CONTRIBUTIONS}

NG: conception, design and analysis of ChIP and PCR experiments and interpretation of data. GL, LM, VV, RS, and CS: designed, performed and analyzed LDH, MTT, and Western Blot

\section{REFERENCES}

Bardai, F. H., and D'Mello, S. R. (2011). Selective toxicity by HDAC3 in neurons: regulation by Akt and GSK3 $\beta$. J. Neurosci. 31, 1746-1751. doi: 10.1523/JNEUROSCI.5704-10.2011

Bolger, T. A., and Yao, T. P. (2005). Intracellular trafficking of histone deacetylase 4 regulates neuronal cell death. J. Neurosci. 25, 9544-9553. doi: 10.1523/JNEUROSCI.1826-05.2005

Ceccatelli, S., Bose, R., Edoff, K., Onishchenko, N., and Spulber, S. (2013). Longlasting neurotoxic effects of exposure to methylmercury during development. J. Intern. Med. 273, 490-497. doi: 10.1111/joim.12045

de Nigris, F., Crudele V., Giovane A., Casamassimi A., Giordano A., Garban H. J., et al. (2010). CXCR4/YY1 inhibition impairs VEGF network and angiogenesis during malignancy. Proc. Natl. Acad. Sci. U.S.A. 107, 14484-14489. doi: 10.1073/pnas.1008256107

Donnici, L., Tiraboschi, E., Tardito, D., Musazzi, L., Racagni, G., and Popoli, M. (2008). Time-dependent biphasic modulation of human BDNF by antidepressants in neuroblastoma cells. BMC Neurosci. 9:61. doi: 10.1186/1471-2202-9-61

Formisano, L., Guida, N., Cocco, S., Secondo, A., Sirabella, R., Ulianich, L., et al. (2011). The repressor element 1-silencing transcription factor is a novel molecular target for the neurotoxic effect of the polychlorinated biphenyl mixture aroclor 1254 in neuroblastoma SH-SY5Y cells. J. Pharmacol. Exp. Ther. 338, 997-1003. doi: 10.1124/jpet.111.181289

Formisano, L., Guida, N., Laudati, G., Boscia, F., Esposito, A., Secondo, A., et al. (2015c). Extracellular signal-related kinase $2 /$ specificity protein $1 /$ specificity protein 3/repressor element-1 silencing transcription factor pathway is involved in Aroclor 1254-induced toxicity in SH-SY5Y neuronal cells. J. Neurosci. Res. 93, 167-177. doi: 10.1002/jnr.23464

Formisano, L., Guida, N., Laudati, G., Mascolo, L., Di Renzo, G., and Canzoniero, L. M. (2015a). MS-275 Inhibits Aroclor 1254-Induced SH-SY5Y Neuronal Cell Toxicity by preventing the formation of the HDAC3/REST complex on the Synapsin-1 Promoter. J Pharmacol. Exp. Ther. 352, 236-243. doi: 10.1124/jpet.114.219345

Formisano, L., Guida, N., Valsecchi, V., Cantile, M., Cuomo, O., Vinciguerra, A., et al. (2015b). Sp3/REST/HDAC1/HDAC2 Complex Represses and Sp1/HIF1/p300 Complex Activates ncxl Gene Transcription, in Brain Ischemia and in Ischemic Brain Preconditioning, by Epigenetic Mechanism. J. Neurosci. 35, 7332-7348. doi: 10.1523/JNEUROSCI.2174-14.2015

Formisano, L., Guida, N., Valsecchi, V., Pignataro, G., Vinciguerra, A., Pannaccione, A., et al. (2013). NCX1 is a new rest target gene: role in cerebral ischemia. Neurobiol. Dis. 50, 76-85. doi: 10.1016/j.nbd.2012.10.010

Fujimura, M., and Usuki, F. (2012). Differing effects of toxicants (methylmercury, inorganic mercury, lead, amyloid $\beta$, and rotenone) on cultured rat cerebrocortical neurons: differential expression of rho proteins associated with neurotoxicity. Toxicol. Sci. 126, 506-514. doi: 10.1093/toxsci/kfr352

Guida, N., Laudati, G., Anzilotti, S., Secondo, A., Montuori, P., Di Renzo, G., et al. (2015a). Resveratrol via sirtuin-1 downregulates RE1-silencing transcription factor (REST) expression preventing PCB-95-induced neuronal cell death. Toxicol. Appl. Pharmacol. 288, 387-398. doi: 10.1016/j.taap.2015.08.010 experiments. LC, GD, and LF: financial support, conception and design of all the experiments and manuscript writing.

\section{FUNDING}

This work was supported by grant: POR Campania FESR 2007-2013 to OCKEY (B25C1300028007) to GD, and PRIN 2015 (2015 BEX2BR) to LF.

\section{ACKNOWLEDGMENTS}

The authors thank Vincenzo Grillo and Carmine Capitale for technical support.

Guida, N., Laudati, G., Anzilotti, S., Sirabella, R., Cuomo, O., Brancaccio, P., et al. (2015b). Methylmercury upregulates RE-1 silencing transcription factor (REST) in SH-SY5Y cells and mouse cerebellum. Neurotoxicology 52, 89-97. doi: 10.1016/j.neuro.2015.11.007

Guida, N., Laudati, G., Galgani, M., Santopaolo, M., Montuori, P., Triassi, M., et al. (2014). Histone deacetylase 4 promotes ubiquitin-dependent proteasomal degradation of Sp3 in SH-SY5Y cells treated with di(2-ethylhexyl)phthalate (DEHP), determining neuronal death. Toxicol. Appl. Pharmacol. 280, 190-198. doi: 10.1016/j.taap.2014.07.014

Guida, N., Laudati, G., Mascolo, L., Cuomo, O., Anzilotti, S., Sirabella, R., et al. (2016). MC1568 Inhibits Thimerosal-Induced Apoptotic cell death by preventing HDAC4 Up-Regulation in Neuronal Cells and in Rat Prefrontal Cortex. Toxicol. Sci. 154, 227-240. doi: 10.1093/toxsci/kfw157

He, D. Y., Neasta, J., and Ron, D. (2010). Epigenetic regulation of BDNF expression via the scaffolding protein RACK1. J. Biol. Chem. 285, 19043-19050. doi: 10.1074/jbc.M110.100693

Hofer, M., Pagliusi, S. R., Hohn, A., Leibrock, J., and Barde, Y. A. (1990). Regional distribution of brain-derived neurotrophic factor mRNA in the adult mouse brain. EMBO J. 9, 2459-2464.

Johar, K., Priya, A., Dhar, S., Liu, Q., and Wong-Riley, M. T. (2013). Neuronspecific specificity protein 4 bigenomically regulates the transcription of all mitochondria- and nucleus-encoded cytochrome c oxidase subunit genes in neurons. J. Neurochem. 127, 496-508. doi: 10.1111/jnc.12433

Johnson, F. O., Yuan, Y., Hajela, R. K., Chitrakar, A., Parsell, D. M., and Atchison, W. D. (2011). Exposure to an environmental neurotoxicant hastens the onset of amyotrophic lateral sclerosis-like phenotype in human $\mathrm{Cu}^{2+} / \mathrm{Zn}^{2+}$ superoxide dismutase 1 G93A mice: glutamate-mediated excitotoxicity. J. Pharmacol. Exp. Ther. 338, 518-527. doi: 10.1124/jpet.110.1 74466

Koppel, I., and Timmusk, T. (2013). Differential regulation of Bdnf expression in cortical neurons by class-selective histone deacetylase inhibitors. Neuropharmacology 75, 106-115. doi: 10.1016/j.neuropharm.2013.07.015

Liu, F., Pore, N., Kim, M., Voong, K. R., Dowling, M., Maity, A., et al. (2006). Regulation of histone deacetylase 4 expression by the SP family of transcription factors. Mol. Biol. Cell 17, 585-597. doi: 10.1091/mbc.E05-08-0775

Lu, T. H., Hsieh, S. Y., Yen, C. C., Wu, H. C., Chen, K. L., Hung, D. Z., et al. (2011). Involvement of oxidative stress-mediated ERK1/2 and p38 activation regulated mitochondria-dependent apoptotic signals in methylmercury-induced neuronal cell injury. Toxicol. Lett. 204, 71-80. doi: 10.1016/j.toxlet.2011.04.013

Myers, G. J., and Davidson, P. W. (1998). Prenatal methylmercury exposure and children: neurologic, developmental, and behavioral research. Environ. Health Perspect. 106(Suppl. 3), 841-847. doi: 10.1289/ehp.98106841

Nagahara, A. H., Merrill, D. A., Coppola, G., Tsukada, S., Schroeder, B. E., Shaked, G. M., et al. (2009). Neuroprotective effects of brain-derived neurotrophic factor in rodent and primate models of Alzheimer's disease. Nat. Med. 15, 331-337. doi: 10.1038/nm.1912

Ni, M., Li, X., Yin, Z., Sidoryk-Wegrzynowicz, M., Jiang, H., Farina, M., et al. (2011). Comparative study on the response of rat primary astrocytes and microglia to methylmercury toxicity. Glia 59, 810-820. doi: 10.1002/glia.21153 
Posser, T., Dunkley, P. R., Dickson, P. W., and Franco, J. L. (2010). Human neuroblastoma cells transfected with tyrosine hydroxylase gain increased resistance to methylmercury-induced cell death. Toxicol. In Vitro 24, 1498-1503. doi: 10.1016/j.tiv.2010.07.015

Qiu, Z., Norflus, F., Singh, B., Swindell, M. K., Buzescu, R., Bejarano, M., et al. (2006). Sp1 is up-regulated in cellular and transgenic models of Huntington disease, and its reduction is neuroprotective. J. Biol. Chem. 281, 16672-16680. doi: 10.1074/jbc.M511648200

Rivieccio, M. A., Brochier, C., Willis, D. E., Walker, B. A., D’Annibale, M. A., McLaughlin, K., et al. (2009). HDAC6 is a target for protection and regeneration following injury in the nervous system. Proc. Natl. Acad. Sci. U.S.A. 106, 19599-19604. doi: 10.1073/pnas.0907935106

Saramäki, A., Diermeier, S., Kellner, R., Laitinen, H., Vaïsänen, S., and Carlberg, C. (2009). Cyclical chromatin looping and transcription factor association on the regulatory regions of the p21 (CDKN1A) gene in response to lalpha,25-dihydroxyvitamin D3. J. Biol. Chem. 284, 8073-8082. doi: 10.1074/jbc.M808090200

Sen, A., Nelson, T. J., and Alkon, D. L. (2015). ApoE4 and Abeta Oligomers Reduce BDNF Expression via HDAC nuclear translocation. J. Neurosci. 35, 7538-7551. doi: 10.1523/JNEUROSCI.0260-15.2015

Shao, Y., Figeys, D., Ning, Z., Mailloux, R., and Chan, H. M. (2015). Methylmercury can induce Parkinson's-like neurotoxicity similar to 1methyl-4- phenylpyridinium: a genomic and proteomic analysis on MN9D dopaminergic neuron cells. J. Toxicol. Sci. 40, 817-828. doi: 10.2131/jts.4 0.817

Sirabella, R., Secondo, A., Pannaccione, A., Molinaro, P., Formisano, L., Guida, N., et al. (2012). ERK1/2, p38, and JNK regulate the expression and the activity of the three isoforms of the $\mathrm{Na}^{+} / \mathrm{Ca}^{2+}$ exchanger, NCX1, NCX2, and NCX3, in neuronal PC12 cells. J. Neurochem. 122, 911-922. doi: 10.1111/j.1471-4159.2012.07838.x

Spulber, S., Rantamäki, T., Nikkilä, O., Castrén, E., Weihe, P., Grandjean, P., et al. (2010). Effects of maternal smoking and exposure to methylmercury on brain-derived neurotrophic factor concentrations in umbilical cord serum. Toxicol. Sci. 117, 263-269. doi: 10.1093/toxsci/kfq216

Suske, G. (1999). The Sp-family of transcription factors. Gene 238, 291-300. doi: 10.1016/S0378-1119(99)00357-1

Tao, X., Finkbeiner, S., Arnold, D. B., Shaywitz, A. J., and Greenberg, M. E. (1998). $\mathrm{Ca}^{2+}$ influx regulates BDNF transcription by a CREB family transcription factor-dependent mechanism. Neuron 20, 709-726. doi: 10.1016/S0896-6273(00)81010-7

Vinciguerra, A., Formisano, L., Cerullo, P., Guida, N., Cuomo, O., Esposito, A., et al. (2014). MicroRNA-103-1 selectively downregulates brain NCX1 and its inhibition by Anti-miRNA ameliorates stroke damage and neurological deficits. Mol. Ther. 22, 1829-1838. doi: 10.1038/mt.2014.113

Volmar, C.-H., and Wahlestedt, C. (2015). Histone deacetylases (HDACs) and brain function. Neuroepigenetics 1, 20-27. doi: 10.1016/j.nepig.2014.10.002

Xia, Z., Dickens, M., Raingeaud, J., Davis, R. J., and Greenberg, M. E. (1995). Opposing effects of ERK and JNK-p38 MAP kinases on apoptosis. Science 270, 1326-1331. doi: 10.1126/science.270.5240.1326

Yuan, H., Denton, K., Liu, L., Li, X. J., Benashski, S., McCullough, L., et al. (2016). Nuclear translocation of histone deacetylase 4 induces neuronal death in stroke. Neurobiol. Dis. 91, 182-193. doi: 10.1016/j.nbd.2016.03.004

Conflict of Interest Statement: The authors declare that the research was conducted in the absence of any commercial or financial relationships that could be construed as a potential conflict of interest.

Copyright (c) 2017 Guida, Laudati, Mascolo, Valsecchi, Sirabella, Selleri, Di Renzo, Canzoniero and Formisano. This is an open-access article distributed under the terms of the Creative Commons Attribution License (CC BY). The use, distribution or reproduction in other forums is permitted, provided the original author(s) or licensor are credited and that the original publication in this journal is cited, in accordance with accepted academic practice. No use, distribution or reproduction is permitted which does not comply with these terms. 